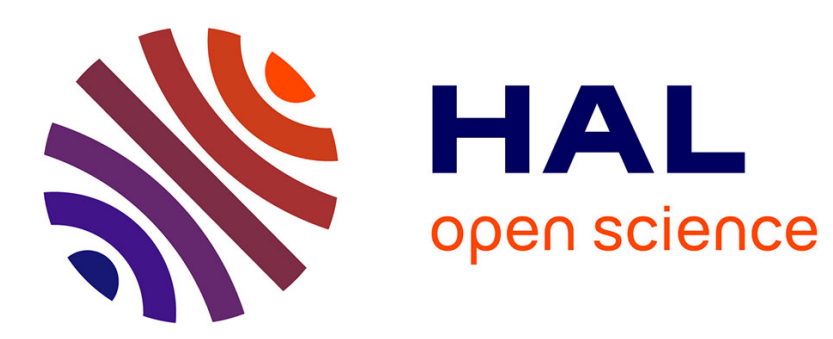

\title{
Effects of magnetic excitations and transitions on vacancy formation: cases of fcc Fe and Ni compared to bcc Fe
}

Kangming Li, Chu Chun Fu, Anton Schneider

\section{- To cite this version:}

Kangming Li, Chu Chun Fu, Anton Schneider. Effects of magnetic excitations and transitions on vacancy formation: cases of fcc Fe and Ni compared to bcc Fe. Physical Review B: Condensed Matter and Materials Physics (1998-2015), 2021, 104 (10), pp.104406. 10.1103/PhysRevB.104.104406 . cea03562009

\section{HAL Id: cea-03562009 https://hal-cea.archives-ouvertes.fr/cea-03562009}

Submitted on 8 Feb 2022

HAL is a multi-disciplinary open access archive for the deposit and dissemination of scientific research documents, whether they are published or not. The documents may come from teaching and research institutions in France or abroad, or from public or private research centers.
L'archive ouverte pluridisciplinaire HAL, est destinée au dépôt et à la diffusion de documents scientifiques de niveau recherche, publiés ou non, émanant des établissements d'enseignement et de recherche français ou étrangers, des laboratoires publics ou privés. 


\title{
Effects of magnetic excitations and transitions on vacancy formation: Cases of fec Fe and Ni compared to bec Fe
}

\author{
Kangming $\mathrm{Li} \odot$ and Chu-Chun Fu॰ \\ Université Paris-Saclay, CEA, Service de Recherches de Métallurgie Physique, F-91191 Gif-sur-Yvette, France \\ Anton Schneider (i) \\ Engineering Physics Department, University of Wisconsin, Madison, Wisconsin 53706, USA
}

(Received 16 June 2021; revised 19 August 2021; accepted 20 August 2021; published 2 September 2021)

\begin{abstract}
Vacancy is one of the most frequent defects in metals. We study the impacts of magnetism on vacancy formation properties in fcc $\mathrm{Ni}$, and in bcc and fcc Fe, via density functional theory (DFT) and effective interaction models combined with Monte Carlo simulations. Overall, the predicted vacancy formation energies and equilibrium vacancy concentrations are in good agreement with experimental data, available only at the high-temperature paramagnetic regime. Effects of magnetic transitions on vacancy formation energies are found to be more important in bcc Fe than in fcc Fe and Ni. The distinct behavior is correlated to the relative roles of longitudinal and transversal spin excitations. At variance with the bcc-Fe case, we note a clear effect of longitudinal spin excitations on the magnetic free energy of vacancy formation in fcc Fe and $\mathrm{Ni}$, leading to its steady variation above the respective magnetic transition temperature. Below the Néel point, such effect in fcc $\mathrm{Fe}$ is comparable but opposite to the one of the transversal excitations. Regarding fcc $\mathrm{Ni}$, although neglecting the longitudinal spin excitations induces an overestimation of the Curie temperature by $220 \mathrm{~K}$, no additional effect is visible below the Curie point. The distinct effects on the three systems are closely linked to DFT predictions of the dependence of vacancy formation energy on the variation of local magnetic-moment magnitudes and orientations.
\end{abstract}

DOI: 10.1103/PhysRevB.104.104406

\section{INTRODUCTION}

Vacancy is one of the simplest and most frequently encountered structural defects in transition-metal systems. Vacancy concentration $[V]$ is a key parameter controlling atomic transport via the vacancy mechanism [1]. The equilibrium monovacancy concentration $[V]_{\text {eq }}$ can be linked with the vacancy formation free energy $G_{f}$ via the expression [2-4]

$$
[V]_{\mathrm{eq}}=\exp \left(-\frac{G_{f}}{k_{B} T}\right),
$$

where $k_{B}$ and $T$ are, respectively, the Boltzmann constant and the absolute temperature, and $G_{f}$ includes all the nonconfigurational entropy contributions (electronic, vibrational, and magnetic) [5]. However, neither $[V]$ nor $G_{f}$ can always be directly measured in experiments. $[V]$ can be determined from differential dilatometry, but in a thermal-vacancy regime, the technique is only applicable at very high temperatures (sometimes near the melting point) due to the limited experimental resolution [6]. Other methods such as electrical resistivity measurement and positron annihilation spectroscopy usually provide the temperature dependence of $[V]$ rather than its absolute value [7]. For pure systems, $G_{f}$ can also be indirectly estimated as $G_{a}-G_{m}$, where $G_{a}$ is the diffusion activation free energy determined from tracer diffusion experiments $[8,9]$, and $G_{m}$ is the vacancy migration free energy coming from resistivity recovery, internal friction, or magnetic aftereffect experiments [10-13].

In magnetic metal systems, several thermodynamic and kinetic properties are affected by thermal magnetic excitations and the magnetic transition. The additional magnetic degree of freedom makes an accurate prediction of those properties more difficult, particularly from an atomisticsimulation point of view. Experimental measurements of selfand solute diffusion coefficients in bcc Fe revealed strong effects of magnetism [9,14-17]. These experimental evidences have motivated various recent theoretical studies [18-24]. However, very little is known about such effects on the vacancy-related properties in other magnetic systems (for example fcc $\mathrm{Fe}$, fcc $\mathrm{Ni}$, and bcc $\mathrm{Cr}$ ) that exhibit important longitudinal spin fluctuations at finite temperatures [25-28].

The thermal magnetic effects can be very system dependent. Here we focus on fcc $\mathrm{Fe}$ and fcc $\mathrm{Ni}$, which are the major constituents for the technologically important austenitic steels. At variation with the bcc-Fe case, experimental data on the vacancy concentration and the vacancy formation free energy are only available for the paramagnetic (PM) states of fcc Fe [29-33] and fcc Ni [6,34-36], due to the low magnetic transition temperatures (67 K for fcc $\mathrm{Fe}$ [37] and $627 \mathrm{~K}$ for fcc Ni [38]). On the theoretical side, previous first principles investigations on the vacancy formation and diffusion properties in the PM regime were often informed by results on the ferromagnetic (FM) state of fcc Ni [39-43] and the 
nonmagnetic (NM) state in the case of fcc Fe [44-46]. It is still an open question whether these results, calculated with ordered or nonmagnetic states, are representative for those in the PM state. For example, the vacancy formation energy obtained for $\mathrm{NM}$ fcc $\mathrm{Fe}$ is $2.37 \mathrm{eV}$, much higher than the experimental values (1.40-1.83 eV [29-33]). More advanced descriptions of PM state properties can be achieved via the disordered local moment (DLM) approach $[47,48]$, the spin-wave method [49], spin dynamics [50-52], spin-lattice dynamics [53,54], and Monte Carlo simulations based on magnetic model Hamiltonians [55-58] (see Ref. [59] for a recent review), etc. Most of these studies $[47-58,60,61]$ only considered defectfree systems. Regarding the vacancy properties in the PM state, most of the investigations addressed bcc Fe [20-24], while there are very few such studies of fcc Fe [62] or fcc $\mathrm{Ni}$. Furthermore, the effects of longitudinal spin fluctuations, which can be important in the PM regime, are often neglected. Taking into account such effects requires, for example in the DLM approach, a more sophisticated statistical treatment combined with constrained local-moment calculations $[25,28,63]$, leading to very CPU-demanding calculations. Finally, the previous theoretical studies in fcc $\mathrm{Fe}$ and $\mathrm{Ni}$ addressed the vacancy properties in the magnetic ground state and the high-temperature ideal PM state, whereas a systematic understanding of the temperature-dependent magnetic effects, which involve simultaneous longitudinal and transversal spin excitations, is missing.

In this study, we intend to provide a detailed analysis of the effects of longitudinal and transversal spin excitations and the magnetic transitions on vacancy formation properties, based on DFT parametrized magnetic effective interaction models (EIMs). The investigations are performed on fcc Fe and fcc $\mathrm{Ni}$, using the EIMs parametrized in this work, and on bcc $\mathrm{Fe}$ with the EIM from a previous study [24]. A systematic comparison of the effects between the three systems is performed.

The paper is organized as follows. We introduce the computational details in Sec. II and demonstrate the accuracy of the EIMs in Sec. III. The vacancy formation properties from first principles calculations and the EIM predictions are discussed in Sec. IV, for various temperature regimes across the magnetic transition. Finally, conclusions are given in Sec. V.

\section{COMPUTATIONAL DETAILS}

The present modeling strategy is as follows. We first performed DFT calculations in fcc Fe and Ni systems, with and without a vacancy (Sec. II A). Then, these results were used to parametrize EIMs (Sec. II B), which were later employed in on-lattice Monte Carlo simulations (Sec. II C), for the study of temperature-dependent vacancy formation properties.

\section{A. DFT calculations}

DFT calculations were performed using the projector augmented wave (PAW) method [64,65] as implemented in the Vienna ab initio simulation package (VASP) code [66-68]. $3 d$ and $4 s$ electrons of $\mathrm{Fe}$ and $\mathrm{Ni}$ atoms were considered as valence electrons. The plane-wave basis cutoff was set to $400 \mathrm{eV}$. The Methfessel-Paxton broadening scheme with a smearing width of $0.1 \mathrm{eV}$ was used [69]. The convergence cut- off for the electronic self-consistency loop was set to $10^{-6} \mathrm{eV}$. The $k$-point grids were adjusted according to the cell size to achieve a $k$-sampling equivalent to a cubic unit cell with a $16^{3}$ shifted grid following the Monkhorst-Pack scheme [70]. Atomic magnetic moments were obtained by an integration of spin-up and spin-down charge densities within the PAW spheres, with a radius of $1.302 \AA$ for $\mathrm{Fe}$ and $1.286 \AA$ for Ni.

We mainly adopted the generalized gradient approximation (GGA) for the exchange-correlation functional in the Perdew-Burke-Ernzerhof (PBE) parametrization [71]. Some additional local density approximation (LDA) calculations were performed for fcc $\mathrm{Ni}$ to examine the effects of the exchange-correlation functional on the vacancy formation energies.

We use the following notations for different magnetic states: NM, FM, and PM denote nonmagnetic, ferromagnetic, and paramagnetic states, respectively; AFS and AFD denote the antiferromagnetic single-layer and double-layer states, respectively; Z1 denotes the magnetic structure consisting of three consecutive spin-up layers and one spin-down layer along one direction. The PM state in DFT is approximated by magnetic special quasirandom structures (mSQS) [59].

DFT calculations were performed with various collinear magnetic structures for fcc $\mathrm{Fe}$ and $\mathrm{Ni}$, and a few noncollinear ones for fcc Fe. For magnetically ordered states (NM, FM, AFS, AFD, and Z1), the atomic positions, the volume, and the cell shape were fully optimized. For other magnetic states with local or global magnetic disorders, the atomic positions were fixed to those in the magnetic collinear ground states (namely AFD Fe and FM Ni) while the volume and the cell shape were optimized.

For the defect-free systems, supercells of various sizes containing up to 128 atoms were used. In total, we considered more than 120 and 60 different magnetic configurations for fcc Fe and Ni, respectively.

For the systems containing a vacancy, we performed calculations with either fully relaxing the magnetic configurations or constraining atomic magnetic moments. In the former case, 108 -site and 128-site supercells were used. They were respectively constructed as $3 \times 3 \times 3$ four-atom face-centered cubic (fcc) unit cells and $4 \times 4 \times 4$ two-atom body-centered tetragonal (bct) unit cells. In the constrained-magnetism case, we used 54-site $(3 \times 3 \times 3$ two-atom unit cells $)$ and 72-site $(3 \times 3 \times 4$ two-atom unit cells) supercells. The vacancy formation energy $E_{f}$ was calculated as

$$
E_{f}=E_{\mathrm{tot}, V}-\frac{N-1}{N} E_{\mathrm{tot}, 0}
$$

with $E_{\mathrm{tot}, V}$ and $E_{\mathrm{tot}, 0}$ the total energies of the systems with and without a vacancy. We verified that $E_{f}$ calculated for a given magnetic configuration but with different supercell sizes differs by at most $5 \mathrm{meV}$. In total, we considered around 140 and 90 different magnetic configurations (containing a vacancy) for fcc $\mathrm{Fe}$ and $\mathrm{Ni}$, respectively.

Vibrational entropies of vacancy formation of the magnetic collinear ground states were calculated within the harmonic approximation in $3 \times 3 \times 3$ supercells using VASP and PHONOPY [72]. They were then added to the vacancy formation magnetic free energies from Monte Carlo simulations. Consequently, the magnon-phonon coupling effects 
$[21,22,73]$ and the anharmonic effects $[5,74,75]$ were neglected. The many-electron effects, which were found to be relevant to the magnetic properties in $\mathrm{Fe}$ according to dynamical mean-field theory calculations [76-78], were not considered in the present study.

\section{B. Effective interaction models (EIM)}

We adopt a model Hamiltonian similar to those used to investigate magnetic properties, phase stability [55-57,61], and vacancy formation and diffusion properties [24,58,79] of Fe-based systems. The Hamiltonians for the present pure systems are as follows:

$$
E_{\mathrm{tot}}=\sum_{i} \sigma_{i} \cdot\left(\epsilon_{i}+A_{i} M_{i}^{2}+B_{i} M_{i}^{4}+\sum_{j} \sigma_{j} \cdot J_{i j} \boldsymbol{M}_{i} \boldsymbol{M}_{j}\right),
$$

where $i$ denotes the $i$ th lattice site, $\sigma_{i}$ is the occupation variable and is equal to $1(0)$ for an occupied (empty) lattice site, $\epsilon_{i}$ is the onsite nonmagnetic parameter, $M_{i}$ is the local magnetic moment, $A_{i}$ and $B_{i}$ are the onsite magnetic parameters controlling the magnitudes of magnetic moments, $J_{i j}$ are the exchange interaction parameter controlling the orientations of magnetic moments, and $\sum_{j}$ is a sum over all the neighboring sites up to the fourth neighbor shell. The Hamiltonian has a generalized Heisenberg form enabling both longitudinal and transversal spin variations. The effects of the presence of a vacancy are included via a dependence of the model parameters on the distance between the $i$ th atom and the vacancy [80], in a similar way as in Refs. [24,58]. Specifically, we consider three sets of values for the model parameters for the cases where the atom is at the first or second nearest neighbor shells or beyond the second nearest neighbor shell of the vacancy. This choice is based on the DFT results showing that the presence of a vacancy modifies mainly the local electronic structure and therefore the local magnetic-moment magnitude of its first and second nearest neighbors. Please note that the present models are only parametrized to consider isolated vacancies.

In this work, we investigate the vacancy formation properties in fcc Fe and $\mathrm{Ni}$, and compare them with those in bcc Fe. For bcc Fe, our previously developed model is used [24]. For fcc $\mathrm{Fe}$ and $\mathrm{Ni}$, we parametrize new EIMs as follows. First, the magnetic parameters of bulk atoms are fitted to energy differences between various magnetic states of defectfree fcc Fe and Ni systems. Then, the magnetic parameters of the atoms in the first and second coordination shells of the vacancy are fitted to the energy differences between different magnetic structures containing a vacancy. Finally, the nonmagnetic parameters are fitted to the vacancy formation energies of AFD Fe and FM Ni (the respective collinear magnetic ground states). The numerical values of the parameters can be found in the Supplemental Material [80].

\section{On-lattice Monte Carlo simulations}

On-lattice spin Monte Carlo (SMC) simulations for fcc Fe and $\mathrm{Ni}$ are performed on fcc supercells consisting of $16^{3}$ cubic unit cells (16 $384 \mathrm{fcc}$ sites) with periodic boundary conditions.
For bcc Fe, we use bcc supercells consisting of $20^{3}$ cubic unit cells (16 000 bcc sites).

The spin system is equilibrated at a given temperature by performing Metropolis SMC steps. At each SMC step, a random variation of the magnetic moment of a randomly chosen atom is attempted with the acceptance probability $\min [1, \exp (\Delta E / \eta)]$. Within the classical statistics, $\eta$ is equal to $k_{B} T$. However, a quantum statistics treatment of spins at low temperatures was shown to be necessary for various magnetic and vacancy properties [24]. As detailed in the Supplemental Material [80], the quantum effects on magnetism can be incorporated through the scaling factor $\eta$ calculated based on the temperature-dependent magnon density of states (mDOS) [54,81]. An accurate determination of temperature-dependent mDOS at the DFT level is beyond the scope of the present study. Instead, we first obtain magnon dispersion relations from the dynamical equation [82] using our model parameters. From them, the ground-state mDOS is calculated and then used to determine the temperaturedependent mDOS based on the quasiharmonic approximation (QHA) [54,81]. The QHA method is known to exaggerate the quantum effects at higher temperatures [81]. For instance, we find a strong exaggeration of the quantum effects in fcc $\mathrm{Ni}$, due to its high magnon energies as compared with the values of bcc and fcc Fe [80,83,84].

In principle, the vacancy formation energy $E_{f}$ can be calculated in a brute-force manner by Eq. (2), using the thermodynamic averages of the total energies for the systems with and without a vacancy. However, it is extremely computationally inefficient to achieve a desired accuracy (e.g., $1 \mathrm{meV}$ ) due to the large fluctuations in the total energies. Furthermore, at finite temperatures, we need to determine the magnetic entropy and free energy of vacancy formation, respectively $S_{f}$ and $G_{f}$. Their calculation from the integration of $E_{f}$ requires a even higher accuracy of the $E_{f}$. In this work, we use the efficient Monte Carlo schemes detailed in the Supplemental Material $[80,85]$ to calculate directly $G_{f}$ with a satisfactory accuracy $(<1 \mathrm{meV})$. Once $G_{f}$ is obtained, $S_{f}$ and $E_{f}$ can be easily evaluated from usual thermodynamic relations. We verify that these schemes yield the same results as those from the aforementioned brute-force method [80], and one of them was already successfully applied to the study of bcc Fe [24].

\section{ACCURACY OF THE EIMs}

As shown in Table I, the energetic hierarchy of several collinear magnetic states of fcc Fe from the EIM is in good agreement with the DFT results. The magnetic ground state of $\mathrm{Fe}$ predicted by the EIM is a spin spiral with

TABLE I. Energies (in meV/atom) of several magnetic states (with respect to AFD) of fcc Fe by the DFT and EIM predictions in this study and the predictions from a previous model [55].

\begin{tabular}{lccccc}
\hline \hline & Noncol-AF & AFD & AFS & FM & NM \\
\hline DFT & - & 0 & 19 & 22 & 82 \\
EIM & -0.5 & 0 & 17 & 29 & 105 \\
EIM in Ref. [55] & -2 & 0 & 0 & 32 & 51 \\
\hline \hline
\end{tabular}




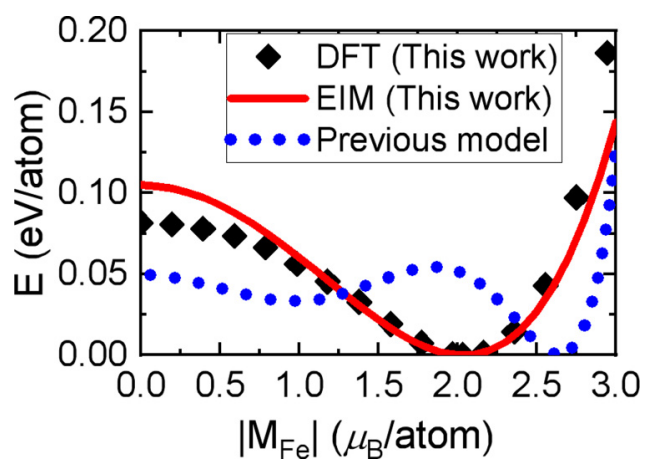

FIG. 1. Energy of AFD fcc Fe as a function of spin magnitude from the DFT and EIM predictions in this study and the prediction from a previous model [55].

$\boldsymbol{q}=\frac{2 \pi}{a}(0,0,0.3)$, which has a slightly lower energy than the AFD state.

Experimentally, the magnetic ground state of fcc $\mathrm{Fe}$ is a spin spiral with $\boldsymbol{q}=\frac{2 \pi}{a}(0.3,0,1)$ [86-88]. First principles calculations also predicted a spin spiral ground state, but with $\boldsymbol{q}=\frac{2 \pi}{a}(0,0,0.6)$ [89-91]. Within the collinear approximation, the ground state is the AFD state [91-94], with $\boldsymbol{q}=$ $\frac{2 \pi}{a}(0,0,0.5)$.

We fit the EIM parameters of fcc Fe on the DFT results of various magnetic states, in particular on the energy of the AFD state as a function of magnetic-moment magnitude (Fig. 1). The latter energy landscape is not well captured by a recent effective interaction model in Ref. [55], which also gives an overestimated equilibrium spin magnitude for the AFD Fe. Note that it is crucial to well reproduce such an energetic landscape, because it dictates the description of longitudinal spin excitations at finite temperatures, which are important in fcc Fe and Ni [26-28]. Furthermore, our DFT results show that the vacancy formation energies are sensitive to the spin magnitudes.

The EIM of pure Ni is also fitted on the DFT results of various collinear states and the energy-versus-moment relation in the FM state. In agreement with the DFT results, the magnetic ground state of Ni predicted by the EIM is FM with a magnetic moment of $0.65 \mu_{\mathrm{B}}$.

\section{RESULTS AND DISCUSSIONS}

In this section, we present and discuss the DFT and EIM predictions of bulk and vacancy formation properties in fcc $\mathrm{Fe}$ and $\mathrm{Ni}$, with a comparison to those in bcc Fe.

\section{A. Magnetic properties of the defect-free bulk systems}

As shown in Table II, the generalized Heisenberg (GH) EIMs reproduce well the experimental Curie temperatures of bcc Fe and fcc $\mathrm{Ni}$, and give a closer prediction of the measured Néel temperature of fcc Fe than that of $450 \mathrm{~K}$ from a previous model [55]. Though the predicted Néel temperature is still somehow overestimated, it is ensured that fcc Fe is already paramagnetic at the room temperature.

It is known that longitudinal spin fluctuations are more significant in fcc Fe and Ni than in bcc Fe [26-28]. This point is also confirmed by the EIM results shown in Fig. 2. Compared
TABLE II. Experimental and calculated Curie temperature of bcc $\mathrm{Fe}$ and fcc Ni and Néel temperature of fcc Fe. GH and $\mathrm{CH}$ denote the generalized Heisenberg (i.e., freely evolving spin magnitudes) and classical Heisenberg (i.e., fixed spin magnitudes) simulations, respectively.

\begin{tabular}{lccc}
\hline \hline System & Expt. & GH & CH \\
\hline bcc Fe & $1044 \mathrm{~K} \mathrm{[38]}$ & $1050 \mathrm{~K}$ & $1080 \mathrm{~K}$ \\
fcc Fe & $67 \mathrm{~K} \mathrm{[37]}$ & $220 \mathrm{~K}$ & $240 \mathrm{~K}$ \\
fcc Ni & $627 \mathrm{~K} \mathrm{[38]}$ & $620 \mathrm{~K}$ & $880 \mathrm{~K}$ \\
\hline \hline
\end{tabular}

to the bcc-Fe case, fcc $\mathrm{Ni}$ and fcc Fe show a stronger variation of the average spin magnitude versus temperature, and a larger dispersion of the spin magnitudes.

Our generalized Heisenberg EIMs naturally account for longitudinal spin fluctuations. To demonstrate the effects of such fluctuations on the magnetic transition temperatures $T_{\mathrm{C}}$, we also perform classical Heisenberg $(\mathrm{CH})$ simulations based on the same EIMs, by constraining the spin magnitudes to the ground-state values. The difference between $T_{\mathrm{C}}^{\mathrm{GH}}$ and $T_{\mathrm{C}}^{\mathrm{CH}}$ is then due to the fact that the temperature-dependent variation of the average spin magnitude, and the dispersion of spin magnitudes are neglected in $\mathrm{CH}$ simulations. The effects of neglecting the variation in the average spin magnitude can be roughly estimated as follows. Since the magnetic transition temperature is proportional to $J_{i j} M^{2}, T_{\mathrm{C}}^{\mathrm{CH}}$ can be estimated as

$$
T_{\mathrm{C}}^{\mathrm{CH}}=T_{\mathrm{C}}^{\mathrm{GH}} \times \frac{M^{2}(0 K)}{M^{2}\left(T_{\mathrm{C}}^{\mathrm{GH}}\right)}
$$

where $M^{2}(0 K) / M^{2}\left(T_{\mathrm{C}}^{\mathrm{GH}}\right)$ according to Fig. 2(a) is equal to $1.07,1.14$ and 1.53 for bcc Fe, fcc Fe and fcc Ni, respectively. These estimations are consistent with the results obtained from $\mathrm{CH}$ simulations (Table II). The actual difference in the transition temperatures between the $\mathrm{GH}$ and $\mathrm{CH}$ simulations is small for bcc and fcc Fe, but it becomes quite large in fcc $\mathrm{Ni}$, showing the necessity of taking into account the temperature variation of spin magnitudes. On the other hand, if we impose the $\mathrm{Ni}$ spin magnitudes (in $\mathrm{CH}$ simulations) to be the average value from the $\mathrm{GH}$ simulations at the $T_{\mathrm{C}}^{\mathrm{GH}}$, the obtained $T_{\mathrm{C}}^{\mathrm{CH}}$ is $60 \mathrm{~K}$ lower than the $T_{\mathrm{C}}^{\mathrm{GH}}$. This suggests a minor but nonnegligible effect of the dispersion of atomic spin magnitudes at a given temperature.
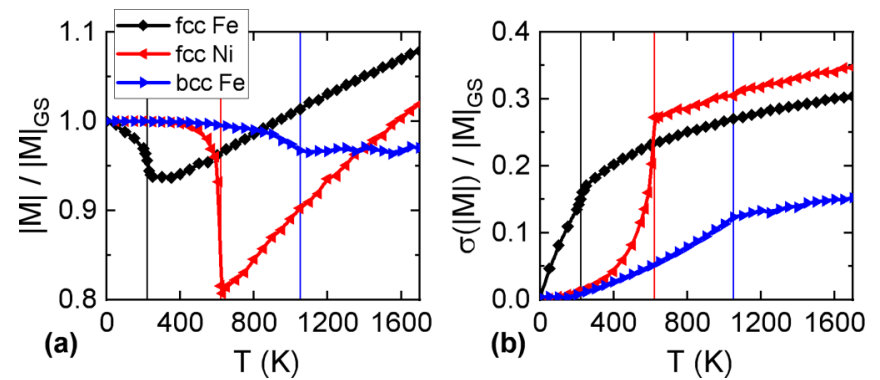

FIG. 2. Variation with temperature of (a) average spin magnitudes and (b) standard deviation of the spin magnitudes, scaled by the respective ground-state moments $|M|_{G S}$. The vertical lines denotes the corresponding magnetic transition temperatures. 
TABLE III. $E_{f}$ (in eV) in different magnetic states computed from DFT. The collinear magnetic ground state values are marked in bold.

\begin{tabular}{lccccc}
\hline \hline System & NM & FM & AFS & AFD & mSQS \\
\hline fcc Fe & 2.37 & 1.85 & 2.00 & $\mathbf{1 . 8 3}$ & 2.04 \\
fcc Ni & 1.38 & $\mathbf{1 . 4 3}$ & 1.47 & 1.53 & 1.52 \\
bcc Fe & 0.57 & $\mathbf{2 . 2 0}$ & 0.94 & 1.86 & 1.59 \\
\hline \hline
\end{tabular}

\section{B. Vacancy formation energies at $0 \mathrm{~K}$ from DFT}

The vacancy formation energies $E_{f}$ in various magnetic states are computed from DFT (Table III). The dispersion in $E_{f}$ among different magnetic states is much larger in bcc $\mathrm{Fe}$ than in fcc $\mathrm{Fe}$ and $\mathrm{Ni}$, suggesting a stronger magnetic effects on $E_{f}$ in bcc Fe. Please note that such dispersions are related to not only the ordering of local magnetic-moment orientations but also the atomic spin magnitudes. The latter can differ by as much as $0.9 \mu_{\mathrm{B}}$ between different magnetic states (excluding the NM one) in bcc and fcc Fe.

To check the dependence of $E_{f}$ on the atomic spin magnitudes $|M|$, we perform the DFT calculations by constraining all the local $|M|$ to the same values. The results for the three systems are shown in Fig. 3.

$E_{f}$ is less dependent on $|M|$ in fcc Ni than in fcc and bcc Fe. In fcc Ni, $E_{f}$ of the FM state is lower than most of other magnetic states with the same $|M|$. Consequently, $E_{f}$ in fcc $\mathrm{Ni}$ at finite temperatures is expected to be larger than that of the FM ground state.

In fcc Fe, $E_{f}$ for all the spin-orientation orderings tends to decrease with increasing $|M|$ for $|M|$ below $2 \mu_{\mathrm{B}}$. Figure 3 also shows that the difference of $E_{f}$ is relatively small between various spin orderings with the same $|M|$, whereas the variation in $E_{f}$ with $|M|$ is relatively large for a given spin ordering. This suggests $E_{f}$ in fcc Fe has a stronger dependence on the atomic spin magnitudes than on the spin orientations. In particular, consider the AFD state and the mSQS. According to the results obtained with optimized magnetic moments (Table III), $E_{f}$ in the AFD state is $0.2 \mathrm{eV}$ lower than that in the mSQS. From Fig. 3, it is clear that $E_{f}$ of the mSQS and the AFD state is very similar for the same $|M|$. Therefore, the difference of $E_{f}$ between the two states in Table III is mainly due to the fact that the optimized $|M|$ is smaller in the
mSQS (on average $\left.1.5 \mu_{\mathrm{B}}\right)$ than in the AFD state $\left(2.0 \mu_{\mathrm{B}}\right)$. This indicates that $E_{f}$ in the mSQS with optimized magnetic moments cannot be taken as the value in the PM state, since longitudinal spin fluctuations in the high temperature PM state lead to an increase in $|M|$.

Contrary to the fcc-Fe case, $E_{f}$ in bcc Fe is much more dependent on the spin-orientation orderings than on the atomic spin magnitudes, as demonstrated in Fig. 3(c). Furthermore, the variation in $|M|$ is also expected to be small in bcc Fe: The optimized magnetic moments in the mSQS are on average $2.05 \mu_{\mathrm{B}}$, rather close to $2.20 \mu_{\mathrm{B}}$ in the FM ground state; it is also shown in the previous section that the thermal longitudinal spin fluctuations in bcc Fe are small. Therefore, the temperature-dependent vacancy formation properties in bcc $\mathrm{Fe}$ are expected to be mainly determined by the arrangement of spin orientations. We note that our EIMs reproduce satisfactorily the above DFT predictions in Table III and Fig. 3.

Finally, we would like to comment on the effects of exchange-correlation functionals on $E_{f}$. In this work, we parametrize the EIMs of fcc Fe and Ni based on the GGA-PBE results. It is well known that GGA describes the bulk (in particular magnetic) properties of Fe better than LDA [91]. For Ni, LDA, and GGA equilibrium lattice parameters $\left(a_{0}\right)$ are $3.416 \AA$ and $3.514 \AA$, respectively, the latter being closer to the experimental value of $3.52 \AA$ [95]. By performing LDA calculations using the GGA $a_{0}$, we verified that adopting LDA results only introduced a constant shift in $E_{f}$ for all the magnetic states compared with GGA values, namely the relative difference in $E_{f}$ between different magnetic states is the same using LDA and GGA. Therefore, the choice of exchangecorrelation functionals influences only the parametrization of the nonmagnetic part of the EIMs. This point will be further discussed for $\mathrm{Ni}$ in Sec. IV C 1.

\section{Vacancy formation properties at finite temperatures from EIM}

We show in Fig. 4 the temperature-dependent vacancy formation properties calculated from $\mathrm{GH}$ simulations for the three systems. Here, $E_{f}^{\mathrm{mag}}$ is the vacancy formation energy, $S_{f}^{\mathrm{mag}}$ is the magnetic contribution to the vacancy formation entropy, and $G_{f}^{\mathrm{mag}}\left(=E_{f}^{\mathrm{mag}}-T S_{f}^{\mathrm{mag}}\right)$ is the vacancy formation magnetic free energy (without the vibrational or other entropic contributions). In the following, we first describe the overall
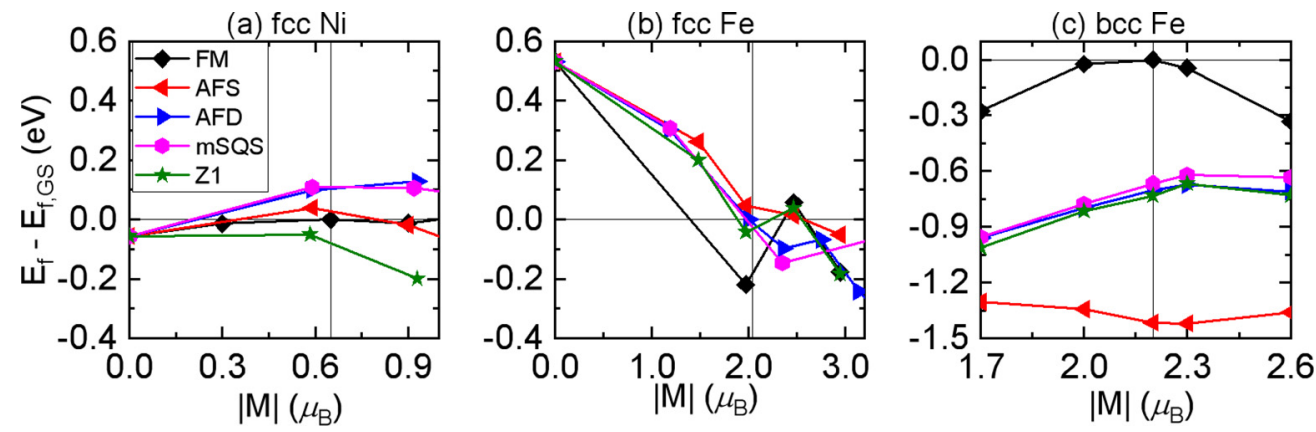

FIG. 3. DFT results of $E_{f}$ in the three systems as a function of constrained local magnetic moment, with respect to $E_{f}$ in the collinear magnetic ground states (1.43 eV for FM fcc Ni, $1.83 \mathrm{eV}$ for AFD fcc Fe and $2.20 \mathrm{eV}$ for FM bcc Fe). The vertical lines denote the spin magnitude of the magnetic ground states. 

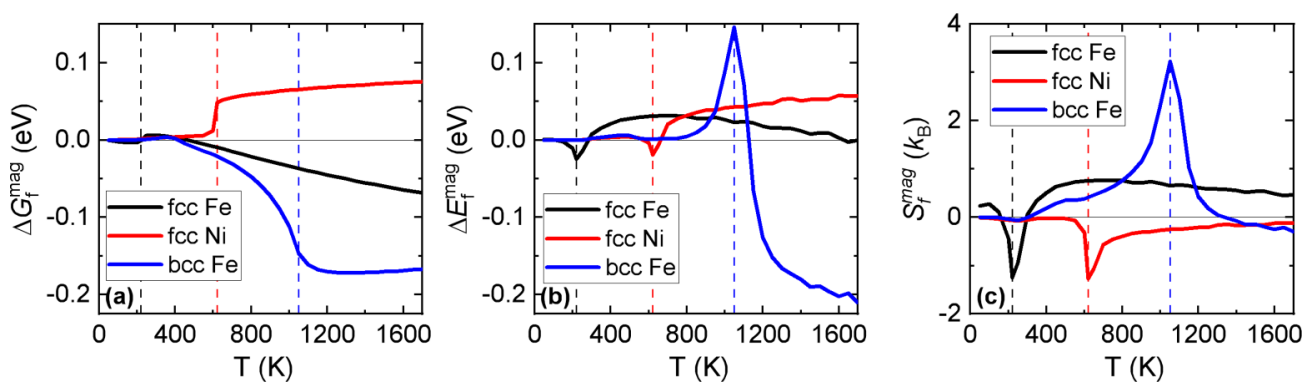

FIG. 4. Magnetic contribution to vacancy formation properties in the three systems calculated from GH simulations (i.e., with both transversal and longitudinal spin fluctuations). $G_{f}^{\mathrm{mag}}$ and $E_{f}^{\mathrm{mag}}$ are given with respect to the ground-state values (1.43 eV for $\mathrm{FM}$ fcc $\mathrm{Ni}$, $1.83 \mathrm{eV}$ for AFD fcc Fe, and $2.20 \mathrm{eV}$ for FM bcc Fe). The vertical lines denote the EIM-predicted magnetic transition temperatures.

behavior versus temperature and compare our results with experimental data. Then, the effects of magnetism in different temperature ranges are analyzed separately.

\section{Overall behavior and comparison with experiments}

We first consider bcc Fe in which the vacancy activation energy strongly depends on the magnetic state as evidenced by experiments $[9,96]$. Theoretical studies reveal that both vacancy formation and migration energies are lower in the fully PM than in the FM state [18-24]. As can be seen in Fig. 4, compared with the FM values, the asymptotic $G_{f}^{\mathrm{mag}}$ and $E_{f}^{\mathrm{mag}}$ are reduced by 0.17 and $0.20 \mathrm{eV}$, respectively.

At variance with the well-known case of bcc $\mathrm{Fe}$, magnetic disorders in fcc $\mathrm{Fe}$ and $\mathrm{Ni}$ do not lead to a strong decrease of $G_{f}^{\mathrm{mag}}$. In the PM region close to the magnetic transition, $\Delta G_{f}^{\mathrm{mag}}=G_{f}^{\mathrm{mag}}(T)-G_{f}^{\mathrm{mag}}(0 K)$ is negligible in fcc Fe but it is positive in fcc Ni. Furthermore, there is a steady variation in $G_{f}^{\text {mag }}$ of fcc $\mathrm{Fe}$ and $\mathrm{Ni}$ in the PM region, which is different from the saturation behavior observed in bcc Fe. These distinct behaviors will be further analyzed in Sec. IV C 3.

The vacancy formation energies in the magnetic ground state and the PM state are compared with other calculations and experiments in Table IV. In bcc Fe, our FM value agrees with the previous calculated results ranging between 2.15$2.23 \mathrm{eV}$ based on the same exchange-correlation functional GGA-PBE [18,22,23], whereas the GGA-PW91 prediction gives a lower value of $1.95 \mathrm{eV}$ [92], or $2.13 \mathrm{eV}$ if using the experimental $a_{0}$ [19]. Our fully PM value is consistent with the previous results, and the dispersion in the calculated values may be due to the different atomic-position relaxation schemes [19-23]. Before comparing with experimental data, it should be noted that the measurement of $E_{f}$ in bcc Fe is extremely sensitive to the presence of interstitial impurities such as carbon, which could lead to an underestimation of $E_{f}$ in earlier studies [96]. Compared with the experimental data in bcc Fe, both our ground-state and PM values are on the higher limit. A very relevant quantity to compare is the difference between the GS and PM energies. It summarizes the overall effect of the magnetic transition, and a cancellation of systematic errors can occur in both calculations and experiments. This difference from our prediction is in good agreement with the result of Schepper et al. [96].

For fcc Fe, our results agree with the previous ab initio predictions $[62,93]$. On the experimental side, the measurements on $E_{f}$ are performed in the PM state. Unlike the bcc-Fe case, the measurement in fcc $\mathrm{Fe}$ is less affected by the presence of impurities [29]. The experimental uncertainty is rather due to its limited temperature window of stability [33]. The predicted $E_{f}$ of PM fcc Fe is consistent with the measured values within the experimental uncertainty. We note that this value is much lower than the one in NM fcc Fe $(2.37 \mathrm{eV})$. The NM state is therefore a poor representative phase to study diffusion properties in fcc Fe, although it has been used in some recent $a b$ initio studies on diffusions in PM fcc Fe [44-46].

To the best of our knowledge, there is no theoretical result for the PM $E_{f}$ and no experimental data for the FM $E_{f}$ in fcc Ni. The experimental $E_{f}$ ranges between $1.5 \mathrm{eV}$ and $1.8 \mathrm{eV}$ (see Ref. [5] and references therein). Smedskjaer et al. [34] suggested that the experimental discrepancies arise from the different analysis methods and the associated assumptions between positron annihilation spectroscopy experiments, and

TABLE IV. Vacancy formation energies (in eV) in the collinear magnetic ground state and the PM state from calculations and experiments. For the PM state, we show both $E_{f}^{\mathrm{mag}}$ and $G_{f}^{\mathrm{mag}}$ (given inside the parentheses) calculated at $1500 \mathrm{~K}$. The small difference between $E_{f}^{\mathrm{mag}}$ and $G_{f}^{\mathrm{mag}}$ comes from the longitudinal spin entropy as explained in Sec. IV C 3.

\begin{tabular}{|c|c|c|c|c|c|c|}
\hline & \multicolumn{2}{|c|}{ bcc Fe } & \multicolumn{2}{|c|}{$\mathrm{fccFe}$} & \multicolumn{2}{|c|}{ fcc Ni } \\
\hline & FM & PM & AFD & PM & FM & PM \\
\hline Other calculations & $1.95-2.24[18-23,92]$ & $1.54-1.98$ [19-23] & $1.82[93]$ & $1.86[62]$ & $1.43[39]$ & \\
\hline Experiments & $\begin{array}{c}2.0 \pm 0.2[96] \\
1.81 \pm 0.1[97] \\
1.60 \pm 0.15[29]\end{array}$ & $\begin{array}{c}1.79 \pm 0.1[96] \\
1.74 \pm 0.1[97] \\
1.53 \pm 0.15[29]\end{array}$ & & $\begin{array}{c}1.40 \pm 0.15[33] \\
1.7 \pm 0.2[32] \\
1.83 \pm 0.14[30]\end{array}$ & & $\begin{aligned} 1.73 & \pm 0.07[36] \\
1.7 & \pm 0.1[35] \\
1.6 & \pm 0.1[35] \\
1.56 & \pm 0.04[98]\end{aligned}$ \\
\hline
\end{tabular}




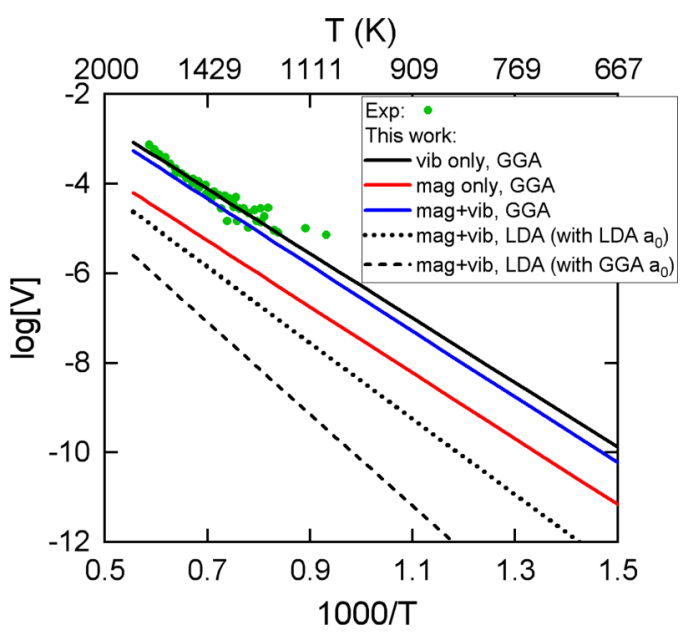

FIG. 5. Calculated $[V]_{\mathrm{eq}}$ as a function of temperature, compared to available experimental data in fcc Ni [98,100-102].

uncontrolled metallurgical variables for other techniques such as positron lifetime spectroscopy. In the light of this suggestion, the recommended value of $E_{f}$ in Ref [6] is $1.79 \mathrm{eV}$. The results from the recent experiments $[35,36,98]$ are shown in Table IV. Though being higher than the recommended value in Ref. [6], our prediction of $E_{f}$ for $\mathrm{PM} \mathrm{Ni}$ is within the uncertainty of the experiments $[33,35,98,99]$, including the most recent one using differential dilatometry [98] to measure directly the equilibrium vacancy concentration.

Indeed, the experimental $E_{f}$ is indirectly deduced from the temperature dependence of the equilibrium vacancy concentrations. Therefore, the latter may allow a more direct comparison. To the best of our knowledge, such measurements on vacancy concentrations have been performed in fcc $\mathrm{Ni}$, but not in bcc and fcc Fe. To determine these concentrations, we have calculated via DFT the vacancy vibrational formation entropy for FM fcc Ni $\left(2.15 k_{B}\right)$ and included it to the magnetic free energy of vacancy formation obtained using the EIM. As shown in Fig. 5, the calculated equilibrium vacancy concentrations of fcc $\mathrm{Ni}$ agree well with the experimental concentrations, which exhibit a larger dispersion at temperatures below $1400 \mathrm{~K}$. We note that $E_{f}$ deduced as the slope of the experimental curve is very sensitive to this dispersion of the experimental data: The estimated $E_{f}$ ranges between 1.5 to $1.6 \mathrm{eV}$ if only data above $1400 \mathrm{~K}$ are considered, and between 1.45 to $1.80 \mathrm{eV}$ if all data are considered.

On the theoretical side, $E_{f}$ of FM Ni is sensitive to the choice of exchange-correlation functional, for instance between the GGA-PBE and the LDA functionals used in DFT calculations [103]. As discussed in Sec. IV B, the choice affects only the EIM prediction of $E_{f}$ of FM Ni but not the temperatures evolution $\Delta G_{f}^{\mathrm{mag}}(T)$. To evaluate the LDAbased prediction of equilibrium vacancy concentrations, we calculated the vacancy formation energy $(1.65 \mathrm{eV})$ and the vacancy vibrational formation entropy $\left(0.43 k_{B}\right)$ of $\mathrm{FM} \mathrm{Ni}$ via DFT using LDA and combined them with $\Delta G_{f}^{\mathrm{mag}}(T)$ predicted by the EIM. Since the experimental $a_{0}$ of Ni is well reproduced by GGA-PBE but underestimated by LDA, we also applied LDA with the GGA-PBE $a_{0}$ to calculate the vacancy formation energy $(2.02 \mathrm{eV})$ and the vacancy vibrational

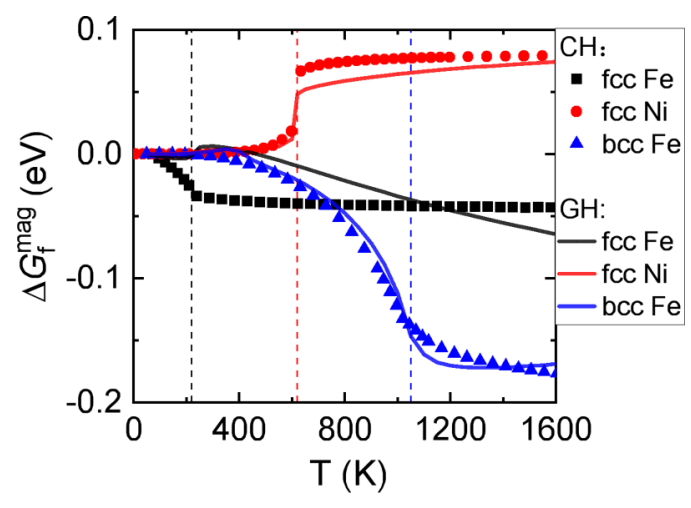

FIG. 6. Comparison of $\Delta G_{f}^{\mathrm{mag}}\left(=G_{f}^{\mathrm{mag}}(T)-G_{f}^{\mathrm{mag}}(0 K)\right)$ as a function of temperature between the $\mathrm{GH}$ and $\mathrm{CH}$ values. For each pure system, the $\mathrm{CH}$ curve is rescaled to have the same magnetic transition temperature as the GH curve. The vertical lines denote the magnetic transition temperatures of the GH curves. $T_{\text {Néel }}$ of fcc Fe is $220 \mathrm{~K}(240 \mathrm{~K})$ from the $\mathrm{GH}(\mathrm{CH})$ calculations; $T_{\text {Curie }}$ of fcc Ni is $620 \mathrm{~K}(880 \mathrm{~K})$ from the $\mathrm{GH}(\mathrm{CH})$ calculations; $T_{\text {Curie }}$ of bcc Fe is $1050 \mathrm{~K}(1080 \mathrm{~K})$ from the $\mathrm{GH}(\mathrm{CH})$ calculations.

formation entropy $\left(0.44 k_{B}\right)$ for $\mathrm{FM} \mathrm{Ni}$. We note the latter is much lower than the GGA-based value $\left(2.15 k_{B}\right)$ and the experimental result (3.3 $\pm 0.5 k_{B}$ [98]). As shown in Fig. 5, the two LDA-based predictions substantially underestimate the experimental equilibrium vacancy concentrations. This may be due to the large vacancy formation energy and the small vacancy vibrational formation entropy obtained with the LDA functional. Therefore, we conclude that the GGA-based prediction is more consistent with the experimental vacancy concentrations.

Finally, we also compare the relative importance of the vibrational and magnetic effects on the equilibrium vacancy concentration in fcc Ni. As can be seen from Fig. 5, neglecting the magnetic effects (namely $G_{f}=G_{f}^{\mathrm{mag}}(0 K)-T S_{f}^{\mathrm{vib}}$ ) changes very little the predicted vacancy concentrations, whereas neglecting the vibrational contribution [namely $G_{f}=$ $\left.G_{f}^{\mathrm{mag}}(T)\right]$ would underestimate the vacancy concentration by one order of magnitude. In fact, for the three systems, the vibrational contribution to the vacancy concentration determination is stronger than the magnetic contribution. The latter is small in fcc Fe and $\mathrm{Ni}$, but it is more significant in bcc Fe as neglecting this can lead to an underestimation of vacancy concentration by up to a factor of 5 .

\section{Vacancy formation below magnetic transitions}

The EIMs for bcc Fe and fcc $\mathrm{Ni}$ predict the same FM ground state as with DFT, therefore the ground state $E_{f}$ for bcc Fe and fcc $\mathrm{Ni}$ are correctly reproduced. For fcc Fe, the collinear magnetic ground state by DFT is AFD, whereas the EIM predicts a spin spiral ground state with a slightly lower energy (by $0.5 \mathrm{meV} /$ atom) than the AFD state. The $E_{f}$ of the spin spiral is found to be slightly higher (by $0.01 \mathrm{eV}$ ) than the AFD value.

The vacancy formation properties below the magnetic transitions can be influenced by the transversal and the longitudinal spin fluctuations, and the effects of two types of fluctuations cannot be easily decoupled. In Fig. 6, we compare 
the temperature evolution of $\Delta G_{f}^{\mathrm{mag}}$ predicted by $\mathrm{GH}$ and $\mathrm{CH}$ simulations.

Below the magnetic transitions, the $\mathrm{CH}$ results (without longitudinal spin fluctuations) follow the same trend as the $\mathrm{GH}$ values for bcc $\mathrm{Fe}$ and fcc $\mathrm{Ni}$, indicating the effects of the longitudinal spin fluctuations are small. This is expected for bcc Fe in which longitudinal spin fluctuations are not significant. For fcc $\mathrm{Ni}$, longitudinal spin fluctuations are rather strong, and the Curie temperature $(880 \mathrm{~K})$ from the $\mathrm{CH}$ simulations is much higher than the $\mathrm{GH}$ value $(620 \mathrm{~K})$. However, the $\mathrm{CH}$ and $\mathrm{GH}$ results of $\Delta G_{f}^{\mathrm{mag}}$ would be similar if they are rescaled to the same Curie temperatures. This is in agreement with the DFT results in Sec. IV B where $E_{f}$ in fcc Ni is found to be rather insensitive to the spin magnitudes, especially for the FM state.

For fcc Fe below the Néel temperature, the $\mathrm{CH}$ values decrease with temperature while the GH values remain nearly unchanged. This suggests that below the magnetic transition in fcc Fe, the transversal spin fluctuations lead to a decrease in $G_{f}^{\mathrm{mag}}$, while the longitudinal spin fluctuations lead to an increase in $G_{f}^{\mathrm{mag}}$. Indeed, the spin magnitudes tend to decrease with temperature before the Néel temperature (Fig. 2), which leads to an increase in $G_{f}^{\mathrm{mag}}$ according to our DFT results in Fig. 3(b).

On the other hand, the spin magnitudes tend to increase with temperature above the Néel temperature, so $G_{f}^{\mathrm{mag}}$ is expected to decrease with temperature according to the DFT results in Fig. 3(b). As shown later, there is a steady decrease in $G_{f}^{\mathrm{mag}}$ with temperature in PM fcc Fe. This demonstrates that the longitudinal spin fluctuations play a dominant role over the transversal ones in the temperature evolution of $G_{f}^{\mathrm{mag}}$ of fcc Fe.

\section{Vacancy formation properties in the PM regime}

As shown in Fig. 6, $G_{f}^{\mathrm{mag}}$ always saturates in the PM region in the $\mathrm{CH}$ simulations. From thermodynamic relations, it can thus be concluded that $E_{f}^{\mathrm{mag}}$ also saturates while $S_{f}^{\mathrm{mag}}$ vanishes at high $T$. Indeed, for $\mathrm{CH}$ models, the magnetic contribution to the vacancy formation energy is zero in the ideal PM state, since the thermodynamic average of the spin-spin correlation $\left\langle\boldsymbol{M}_{i} \boldsymbol{M}_{j}\right\rangle$ is zero in the ideal PM state.

The steady variation in $G_{f}^{\mathrm{mag}}$ in the PM region in Fig. 4(a) is thus related to the longitudinal spin fluctuations and is observed only with the GH models. This behavior can be quantitatively understood as follows. In the ideal PM region where the spin-spin correlations are negligible, the magnetic Hamiltonian can be approximated by a simplified form, retaining only the onsite terms, namely

$$
E_{\mathrm{tot}}^{\mathrm{mag}}=\sum_{i} e_{i}^{\mathrm{mag}}=\sum_{i} A_{i} M_{i}^{2}+B_{i} M_{i}^{4}
$$

where $e_{i}^{\mathrm{mag}}=A_{i} M_{i}^{2}+B_{i} M_{i}^{4}$ is the onsite magnetic energy for the $i$ th atom. The partition function can be expressed as

$$
\begin{aligned}
Z_{\mathrm{tot}} & =\int e^{-\beta E_{\mathrm{tot}}^{\mathrm{mag}}} d \boldsymbol{M}_{1} \ldots d \boldsymbol{M}_{N}=\prod_{i} z_{i} \\
z_{i} & =\int e^{-\beta e_{i}^{\mathrm{mag}}} d \boldsymbol{M}_{i} .
\end{aligned}
$$
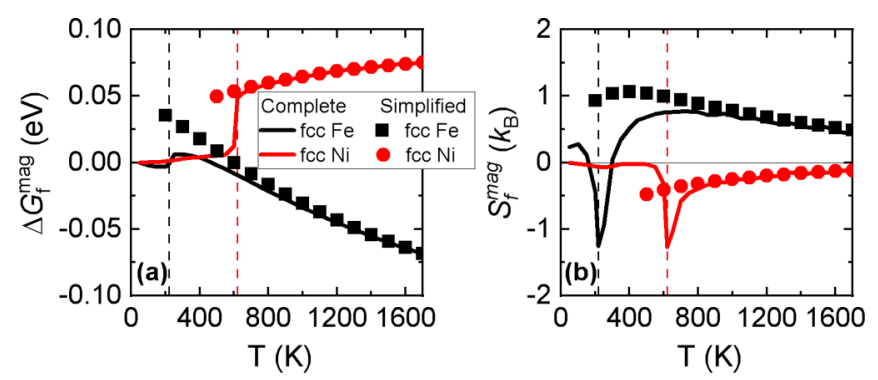

FIG. 7. (a) $\Delta G_{f}^{\mathrm{mag}}$ and (b) $S_{f}^{\mathrm{mag}}$ predicted from the complete and simplified EIMs. The vertical lines denote the EIM-predicted magnetic transition temperatures.

The total magnetic energy of the system can then be calculated as

$$
\begin{aligned}
G_{\mathrm{tot}}^{\mathrm{mag}} & =-k_{B} T \cdot \ln Z_{\mathrm{tot}}=\sum_{i} g_{i}^{\mathrm{mag}} \\
g_{i}^{\mathrm{mag}} & =-k_{B} T \ln z_{i},
\end{aligned}
$$

where $g_{i}^{\text {mag }}$ is the magnetic free energy of the $i$ th atom. In our EIMs, there are three sets of the onsite parameters for each pure system depending on the distance from the vacancy. Consequently, there are three possible values of $g_{i}^{\mathrm{mag}}$ for a pure system containing a vacancy. We use the subscript $i$ equal to 0 , 1 , and 2 to represent the bulk atoms and those in the first and second neighboring shells of the vacancy, respectively. The vacancy formation free energy can then be expressed as

$$
\begin{aligned}
G_{f}^{\mathrm{mag}} & =G_{\mathrm{tot}, V}^{\mathrm{mag}}-\frac{N-1}{N} G_{\mathrm{tot}, 0}^{\mathrm{mag}} \\
& =\sum_{i=1,2} n_{i} \cdot\left(g_{i}^{\mathrm{mag}}-g_{0}^{\mathrm{mag}}\right),
\end{aligned}
$$

where $n_{i}$ is the coordination number of the $i$ th shell.

The simplified EIMs allow a direct numerical evaluation of $z_{i}$ and thus all the subsequent quantities. It should be noted that even though the contribution of transversal spin fluctuations to the averaged total energy is negligible in the PM state, its contribution to the total entropy is not zero. This is fully taken into account in Eq. (7), where it is equally possible for $\boldsymbol{M}_{i}$ to take any direction, as expected for the perfectly PM state. In this way, the transversal part of the entropy per atom is maximized and the same for all the atoms independent of their distances from the vacancy. This entropic contribution is thus canceled out in Eq. (10).

The results of $G_{f}^{\mathrm{mag}}$ and $S_{f}^{\mathrm{mag}}$ are compared between the complete and the simplified EIMs for fcc Fe and Ni in Fig. 7. In the PM region, $G_{f}^{\mathrm{mag}}$ and $S_{f}^{\mathrm{mag}}$ using the simplified EIMs converge to those from the complete EIMs, confirming that the simplified EIMs are very good representations of the complete ones at high temperatures. The different variation trends in $G_{f}^{\mathrm{mag}}$ between fcc Fe and Ni are related to the signs of $S_{f}^{\mathrm{mag}}$. This can also be understood with the simplified Hamiltonians that contain only the onsite magnetic energy $A_{i} M_{i}^{2}+B_{i} M_{i}^{4}$. As shown in Fig. 8, for a given temperature (e.g., $1000 \mathrm{~K}$ as indicated in the figure), the atoms around the vacancy can explore a larger interval of $|M|$, and thus has a larger entropy than the bulk atoms in fcc Fe, while the case is reversed for fcc Ni. 


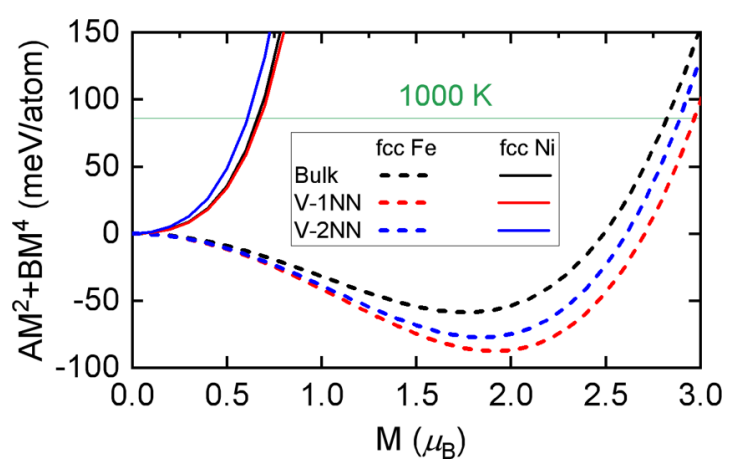

FIG. 8. Onsite magnetic energies $A_{i} M_{i}^{2}+B_{i} M_{i}^{4}$ for the bulk atoms and first and second nearest neighbors of a vacancy.

The current analysis can provide insights into the impact of longitudinal spin variations on vacancy formation at high temperatures. That is, due to the difference of spin magnitudes in the PM state between the atoms near the vacancy and in the bulk, a steady variation of $G_{f}^{\mathrm{mag}}$ with temperature is expected, which is at variance with the predictions from classical Heisenberg models. In this case, it is also nontrivial to apply the well-known Ruch model [104], which proposes

$$
\frac{G_{f}^{\mathrm{mag}}(T)-G_{f}^{\mathrm{mag}}(0 K)}{G_{f}^{\mathrm{mag}}(P M)-G_{f}^{\mathrm{mag}}(0 K)}=1-S^{2},
$$

where $S$ is the magnetic long-range order parameter, and $G_{f}^{\mathrm{mag}}(P M)$ is the vacancy formation magnetic free energy in the ideal PM state. $G_{f}^{\mathrm{mag}}(P M)$ can be taken as the saturated value for bcc Fe, but the definition of $G_{f}^{\mathrm{mag}}(P M)$ is ambiguous for fcc Fe and Ni in which $G_{f}^{\mathrm{mag}}(T)$ does not saturate at high temperatures.

\section{Impact of magnetic transitions on vacancy formation}

It has been shown in Fig. 4 that the effect of the magnetic transition is much stronger in bcc Fe than in fcc Fe and Ni. This is mainly related to the change in the exchange interaction energy across the magnetic transitions. To illustrate this, we begin with the $\mathrm{CH}$ models where $J_{i j}$ are the same for the atoms in the bulk and near the vacancy, and we focus on the vacancy formation energy $E_{f}^{\mathrm{mag}}$ for simplicity. In this case, the exchange interaction energy $\boldsymbol{J}_{i j} \boldsymbol{M}_{i} \boldsymbol{M}_{j}$ does not contribute to $E_{f}^{\text {mag }}$ in the ideal fully PM state. In the magnetic ground state, this contribution to $E_{f}^{\mathrm{mag}}$ is equal to half the ground-state exchange interaction energy per atom. Therefore, the variation in $E_{f}^{\mathrm{mag}}$ across the magnetic transition is equal to half the ground-state exchange interaction energy per atom. The latter value is expected to be larger in bcc Fe than in fcc Fe and $\mathrm{Ni}$ in view of the DFT results $[94,105]$, which show that the energy difference between various magnetic states is more significant in bcc Fe than in fcc Fe and Ni. For a quantitative comparison, we show in Table $\mathrm{V}$ the different contributions to the vacancy formation energies of the three systems. Although there is a compensating variation between the onsite (longitudinal) and exchange (transversal) contributions in the three systems, the latter contribution is much stronger in bcc Fe than in fcc Fe and $\mathrm{Ni}$.
TABLE V. Contributions of different terms in the Hamiltonians to the vacancy formation energy in the magnetic ground state and the PM state, expressed in eV. The PM value is obtained at $1500 \mathrm{~K}$ where the transversal contribution is negligible in all three systems.

\begin{tabular}{|c|c|c|c|c|c|c|}
\hline \multirow[b]{2}{*}{ Contribution } & \multicolumn{2}{|c|}{ bcc Fe } & \multicolumn{2}{|c|}{ fcc Fe } & \multicolumn{2}{|c|}{ fcc Ni } \\
\hline & GS & PM & GS & PM & GS & PM \\
\hline $\mathrm{NM} \epsilon_{i}$ & 2.00 & 2.00 & 2.37 & 2.37 & 1.43 & 1.43 \\
\hline Onsite $A_{i} M_{i}^{2}+B_{i} M_{i}^{4}$ & -0.07 & 0.00 & -0.47 & -0.53 & 0.14 & 0.06 \\
\hline Exchange $J_{i j} \boldsymbol{M}_{i} \boldsymbol{M}_{j}$ & 0.27 & $\approx 0$ & -0.07 & $\approx 0$ & -0.13 & $\approx 0$ \\
\hline Total & 2.20 & 2.00 & 1.84 & 1.85 & 1.43 & 1.48 \\
\hline
\end{tabular}

\section{CONCLUSION}

We investigated magnetic effects on vacancy formation properties in fcc $\mathrm{Ni}$, and in bcc and fcc $\mathrm{Fe}$. We performed density functional theory calculations and Monte Carlo simulations with effective interaction models.

DFT calculations were performed for a large set of magnetic configurations of the three systems, with or without a vacancy. These results reveal a dispersion of the vacancy formation energies $E_{f}$ among various magnetic states, which is larger in bcc and fcc Fe than in fcc Ni. The dispersion is related not only to the ordering of spin orientations but also to the spin magnitudes. We have shown that the spin ordering has a dominant influence on the vacancy formation energy in bcc $\mathrm{Fe}$, while this formation energy in fcc Fe is more dependent on the spin magnitudes; in fcc Ni both types of effects are small.

The DFT-parametrized models, with a generalizedHeisenberg form, were used in Monte Carlo simulations enabling the thermal fluctuations of spin orientations and magnitudes. Regarding magnetic properties of the defect-free systems, the Monte Carlo results reveal that the longitudinal spin fluctuations are more significant in fcc $\mathrm{Fe}$ and $\mathrm{Ni}$ than in bcc Fe. In particular for fcc $\mathrm{Ni}$, neglecting the temperature evolution of spin magnitudes leads to an overestimation by $220 \mathrm{~K}$ of the Curie point. Based on an efficient Monte Carlo scheme, the vacancy formation energy, magnetic entropy, and magnetic free energy were determined as functions of temperature for the three systems.

Overall, the predicted vacancy formation energies and equilibrium vacancy concentrations are in good agreement with the experimental data, which are available only at high temperatures. Concerning the determination of the equilibrium vacancy concentration, the vibrational entropy has a stronger contribution than the magnetic one for all three systems. But the latter is non-negligible in bcc $\mathrm{Fe}$, as neglecting this contribution leads to an underestimation of the equilibrium vacancy concentration by a factor of 5 .

The overall impact of the magnetic transition on the vacancy formation properties are found to be more significant in bcc Fe than in fcc Fe and Ni. The substantial decrease of the vacancy formation energy from the ferromagnetic to the paramagnetic regime in bcc Fe is mainly due to the transversal rather than the longitudinal spin excitations. This is coherent with the strong dependence of the formation energy on the spin-orientation ordering in bcc $\mathrm{Fe}$ as predicted by DFT. 
Consistently, a weaker dependence of the vacancy formation energy on the spin ordering in fcc $\mathrm{Fe}$ and $\mathrm{Ni}$ leads to a smaller variation of the vacancy properties below and above the magnetic transition.

We note a significant effect of longitudinal spin excitations on the magnetic free energy of vacancy formation in fcc Fe, resulting in its steady decrease above the Néel point. Also, below its Néel point, such effect is comparable but opposite to that of the transversal excitations. These are consistent with the DFT results in fcc Fe, which demonstrate a stronger dependence of vacancy formation energy on the spin magnitude rather than the spin ordering. Interestingly, it is noted that the predicted vacancy formation energy in the paramagnetic state is close to the AFD-state value, but it is $0.52 \mathrm{eV}$ lower than the formation energy obtained with nonmagnetic fcc Fe. Although the latter has been used in some recent studies on diffusion properties in $\mathrm{fcc} \mathrm{Fe}$, our results suggest that the paramagnetic fcc Fe is better represented by the AFD state than the nonmagnetic state. Regarding the vacancy formation in fcc $\mathrm{Ni}$, the transversal spin excitations just below the Curie temperature lead to a sudden increase of the vacancy formation magnetic free energy, while the longitudinal spin fluctuations above the Curie temperature lead to a steady increase of this quantity.

The cases of fcc Fe and Ni reveal a relevant effect of longitudinal spin excitations on the vacancy formation properties, which cannot be well captured by a classical Heisenberg model. Also, at variance with the bcc-Fe case, the widely used Ruch model cannot be applied in these systems to predict the temperature evolution of energetics of vacancy formation.

\section{ACKNOWLEDGMENTS}

This work was performed using DARI-GENCI resources under the A0090906020 project, and the CINECAMARCONI supercomputer within the SISTEEL project. The research leading to these results has been partially carried out in the framework of EERA Joint Program for Nuclear Materials and is partly funded by the European Commission HORIZON 2020 Framework Programme under Grant Agreement No. 755269. K.L. is supported by the CEA NUMERICS program, which has received funding from the European Union's Horizon 2020 research and innovation program under the Marie Skłodowska-Curie Grant Agreement No. 800945.
[1] H. Mehrer, Diffus. Solids, Springer Series in Solid-State Sciences (Springer Berlin Heidelberg, Berlin, Heidelberg, 2007), pp. 1-246, Vol. 155.

[2] J. Rogal, S. V. Divinski, M. W. Finnis, A. Glensk, J. Neugebauer, J. H. Perepezko, S. Schuwalow, M. H. F. Sluiter, and B. Sundman, Phys. Status Solidi 251, 97 (2014).

[3] A. V. Ruban, Phys. Rev. B 93, 134115 (2016).

[4] D. Morgan and Y. Zhang, Phys. Rev. B 101, 136101 (2020).

[5] Y. Gong, B. Grabowski, A. Glensk, F. Körmann, J. Neugebauer, and R. C. Reed, Phys. Rev. B 97, 214106 (2018).

[6] H. Ullmaier, P. Ehrhart, P. Jung, and H. Schultz, At. Defects Met., edited by H. Ullmaier, Landolt-Börnstein - Group III Condensed Matter (Springer-Verlag, Berlin,Heidelberg, 1991), Vol. 25.

[7] R. Siegel, J. Nucl. Mater. 69-70, 117 (1978).

[8] N. Peterson, J. Nucl. Mater. 69-70, 3 (1978).

[9] Y. Iijima, K. Kimura, and K. Hirano, Acta Metall. 36, 2811 (1988).

[10] O. Seydel, G. Frohberg, and H. Wever, Phys. Status Solidi 144, 69 (1994).

[11] M. S. Blanter, I. S. Golovin, H. Neuhäuser, and H.-R. Sinning, Internal Friction in Metallic Materials, Springer Series in Materials Science (Springer Berlin Heidelberg, Berlin, Heidelberg, 2007), Vol. 90.

[12] M. Wutting and H. Birnbaum, J. Phys. Chem. Solids 27, 225 (1966).

[13] J. Verdone, P. Moser, W. Chambron, J. Johansson, P. Hautojärvi, and A. Vehanen, J. Magn. Magn. Mater. 19, 296 (1980).

[14] C.-G. Lee, Y. Iijima, T. Hiratani, and K.-i. Hirano, Mater. Trans. JIM 31, 255 (1990).

[15] Y. Iijima, K. Kimura, C.-G. Lee, and K.-i. Hirano, Mater. Trans. JIM 34, 20 (1993).
[16] H. Nitta, T. Yamamoto, R. Kanno, K. Takasawa, T. Iida, Y. Yamazaki, S. Ogu, and Y. Iijima, Acta Mater. 50, 4117 (2002).

[17] S. Takemoto, H. Nitta, Y. Iijima, and Y. Yamazaki, Philos. Mag. 87, 1619 (2007).

[18] S. Huang, D. L. Worthington, M. Asta, V. Ozolins, G. Ghosh, and P. K. Liaw, Acta Mater. 58, 1982 (2010).

[19] H. Ding, V. I. Razumovskiy, and M. Asta, Acta Mater. 70, 130 (2014).

[20] N. Sandberg, Z. Chang, L. Messina, P. Olsson, and P. Korzhavyi, Phys. Rev. B 92, 184102 (2015).

[21] H. Wen and C. H. Woo, J. Nucl. Mater. 470, 102 (2016).

[22] D. Gambino and B. Alling, Phys. Rev. B 98, 064105 (2018).

[23] O. Hegde, M. Grabowski, X. Zhang, O. Waseda, T. Hickel, C. Freysoldt, and J. Neugebauer, Phys. Rev. B 102, 144101 (2020).

[24] A. Schneider, C.-C. Fu, F. Soisson, and C. Barreteau, Phys. Rev. Lett. 124, 215901 (2020).

[25] Z. Dong, W. Li, S. Schönecker, S. Lu, D. Chen, and L. Vitos, Phys. Rev. B 92, 224420 (2015).

[26] A. V. Ruban and M. Dehghani, Phys. Rev. B 94, 104111 (2016).

[27] A. V. Ruban, S. Khmelevskyi, P. Mohn, and B. Johansson, Phys. Rev. B 75, 054402 (2007).

[28] Z. Dong, W. Li, D. Chen, S. Schönecker, M. Long, and L. Vitos, Phys. Rev. B 95, 054426 (2017).

[29] H.-E. Schaefer, K. Maier, M. Weller, D. Herlach, A. Seeger, and J. Diehl, Scr. Metall. 11, 803 (1977).

[30] C. W. Schulte, J. L. Campbell, and J. A. Jackman, Appl. Phys. 16, 29 (1978).

[31] K. Maier, H. Metz, D. Herlach, and H. E. Schaefer, J. Nucl. Mater. 69, 589 (1978).

[32] S. M. Kim and W. J. Buyers, J. Phys. F 8, L103 (1978).

[33] H. Matter, J. Winter, and W. Triftshäuser, Appl. Phys. 20, 135 (1979). 
[34] L. C. Smedskjaer, M. J. Fluss, D. G. Legnini, M. K. Chason, and R. W. Siegel, J. Phys. F 11, 2221 (1981).

[35] T. Troev, C. Angelov, and I. Mincov, Phys. Lett. A 138, 65 (1989).

[36] J. Wolff, M. Franz, J. E. Kluin, and D. Schmid, Acta Mater. 45, 4759 (1997).

[37] A. Onodera, Y. Tsunoda, N. Kunitomi, O. A. Pringle, R. M. Nicklow, and R. M. Moon, Phys. Rev. B 50, 3532 (1994).

[38] J. M. Leger, C. Loriers-Susse, and B. Vodar, Phys. Rev. B 6, 4250 (1972).

[39] J. D. Tucker, R. Najafabadi, T. R. Allen, and D. Morgan, J. Nucl. Mater. 405, 216 (2010).

[40] R. Nazarov, T. Hickel, and J. Neugebauer, Phys. Rev. B 82, 224104 (2010).

[41] C. Z. Hargather, S.-L. Shang, Z.-K. Liu, and Y. Du, Comput. Mater. Sci. 86, 17 (2014).

[42] S. Schuwalow, J. Rogal, and R. Drautz, J. Phys. Condens. Matter 26, 485014 (2014).

[43] C. Z. Hargather, S. L. Shang, and Z. K. Liu, Acta Mater. 157, 126 (2018).

[44] T. Tsuru and Y. Kaji, J. Nucl. Mater. 442, S684 (2013).

[45] Y. Zeng and K. Bai, J. Alloys Compd. 624, 201 (2015).

[46] H. Wang, X. Gao, H. Ren, S. Chen, and Z. Yao, J. Phys. Chem. Solids 112, 153 (2018).

[47] B. L. Gyorffy, A. J. Pindor, J. Staunton, G. M. Stocks, and H. Winter, J. Phys. F 15, 1337 (1985).

[48] B. Alling, F. Körmann, B. Grabowski, A. Glensk, I. A. Abrikosov, and J. Neugebauer, Phys. Rev. B 93, 224411 (2016).

[49] A. V. Ruban and V. I. Razumovskiy, Phys. Rev. B 85, 174407 (2012).

[50] V. Antropov, Phys. Rev. B 72, 140406(R) (2005).

[51] P.-W. Ma and S. L. Dudarev, Phys. Rev. B 86, 054416 (2012).

[52] J. B. J. Chapman, P.-W. Ma, and S. L. Dudarev, Phys. Rev. B 99, 184413 (2019).

[53] P.-W. Ma, C. H. Woo, and S. L. Dudarev, Phys. Rev. B 78, 024434 (2008).

[54] C. H. Woo, H. Wen, A. A. Semenov, S. L. Dudarev, and P.-W. Ma, Phys. Rev. B 91, 104306 (2015).

[55] M. Y. Lavrentiev, J. S. Wróbel, D. Nguyen-Manh, and S. L. Dudarev, Phys. Chem. Chem. Phys. 16, 16049 (2014).

[56] M. Yu. Lavrentiev, C.-C. Fu, and F. Soisson, J. Magn. Magn. Mater. 506, 166763 (2020).

[57] V. T. Tran, C. C. Fu, and A. Schneider, Comput. Mater. Sci. 183, 109906 (2020).

[58] A. Schneider, C.-C. Fu, O. Waseda, C. Barreteau, and T. Hickel, Phys. Rev. B 103, 024421 (2021).

[59] I. Abrikosov, A. Ponomareva, P. Steneteg, S. Barannikova, and B. Alling, Curr. Opin. Solid State Mater. Sci. 20, 85 (2016).

[60] P.-W. Ma, S. L. Dudarev, and C. H. Woo, Phys. Rev. B 85, 184301 (2012).

[61] M. Y. Lavrentiev, J. S. Wróbel, D. Nguyen-Manh, S. L. Dudarev, and M. G. Ganchenkova, J. Appl. Phys. 120, 043902 (2016).

[62] V. I. Razumovskiy, A. Reyes-Huamantinco, P. Puschnig, and A. V. Ruban, Phys. Rev. B 93, 054111 (2016).

[63] D. Gambino, M. Arale Brännvall, A. Ehn, Y. Hedström, and B. Alling, Phys. Rev. B 102, 014402 (2020).

[64] P. E. Blöchl, Phys. Rev. B 50, 17953 (1994).

[65] G. Kresse and D. Joubert, Phys. Rev. B 59, 1758 (1999).
[66] G. Kresse and J. Hafner, Phys. Rev. B 47, 558 (1993).

[67] G. Kresse and J. Furthmüller, Comput. Mater. Sci. 6, 15 (1996).

[68] G. Kresse and J. Furthmüller, Phys. Rev. B 54, 11169 (1996).

[69] M. Methfessel and A. T. Paxton, Phys. Rev. B 40, 3616 (1989).

[70] H. J. Monkhorst and J. D. Pack, Phys. Rev. B 13, 5188 (1976).

[71] J. P. Perdew, K. Burke, and M. Ernzerhof, Phys. Rev. Lett. 77, 3865 (1996).

[72] A. Togo and I. Tanaka, Scr. Mater. 108, 1 (2015).

[73] F. Körmann, A. A. H. Breidi, S. L. Dudarev, N. Dupin, G. Ghosh, T. Hickel, P. Korzhavyi, J. A. Muñoz, and I. Ohnuma, Phys. Status Solidi Basic Res. 251, 53 (2014).

[74] B. Grabowski, L. Ismer, T. Hickel, and J. Neugebauer, Phys. Rev. B 79, 134106 (2009).

[75] A. Glensk, B. Grabowski, T. Hickel, and J. Neugebauer, Phys. Rev. X 4, 011018 (2014).

[76] P. A. Igoshev, A. V. Efremov, A. I. Poteryaev, A. A. Katanin, and V. I. Anisimov, Phys. Rev. B 88, 155120 (2013).

[77] L. V. Pourovskii, J. Mravlje, M. Ferrero, O. Parcollet, and I. A. Abrikosov, Phys. Rev. B 90, 155120 (2014).

[78] A. A. Katanin, A. S. Belozerov, and V. I. Anisimov, Phys. Rev. B 98, 045138 (2018).

[79] A. Schneider, Interplay between magnetism, thermodynamics and diffusion in bcc Fe-Mn alloys: from first principles to finite temperatures, Ph.D. thesis, Université Paris-Saclay (2019).

[80] See Supplemental Material at http://link.aps.org/ supplemental/10.1103/PhysRevB.104.104406 for the details of the model parametrizations, quantum treatment of spins, and the Monte Carlo schemes to calculate the vacancy formation properties.

[81] L. Bergqvist and A. Bergman, Phys. Rev. Materials 2, 013802 (2018).

[82] X. Wu, Z. Liu, and T. Luo, J. Appl. Phys. 123, 085109 (2018).

[83] H. A. Mook and D. M. Paul, Phys. Rev. Lett. 54, 227 (1985).

[84] O. Grotheer, C. Ederer, and M. Fähnle, Phys. Rev. B 63, 100401(R) (2001).

[85] R. W. Zwanzig, J. Chem. Phys. 22, 1420 (1954).

[86] Y. Tsunoda, J. Phys.: Condens. Matter 1, 10427 (1989).

[87] Y. Tsunoda, Y. Nishioka, and R. Nicklow, J. Magn. Magn. Mater. 128, 133 (1993).

[88] D. Qian, X. F. Jin, J. Barthel, M. Klaua, and J. Kirschner, Phys. Rev. Lett. 87, 227204 (2001).

[89] M. Uhl, L. M. Sandratskii, and J. Kübler, J. Magn. Magn. Mater. 103, 314 (1992).

[90] V. M. García-Suárez, C. M. Newman, C. J. Lambert, J. M. Pruneda, and J. Ferrer, J. Phys.: Condens. Matter 16, 5453 (2004).

[91] R. Soulairol, C. C. Fu, and C. Barreteau, J. Phys.: Condens. Matter 22, 295502 (2010).

[92] C. Domain and C. S. Becquart, Phys. Rev. B 65, 024103 (2001).

[93] T. P. C. Klaver, D. J. Hepburn, and G. J. Ackland, Phys. Rev. B 85, 174111 (2012).

[94] K. Li and C.-C. Fu, Phys. Rev. Materials 4, 023606 (2020).

[95] J. Bandyopadhyay and K. Gupta, Cryogenics (Guildf). 17, 345 (1977).

[96] L. De Schepper, D. Segers, L. Dorikens-Vanpraet, M. Dorikens, G. Knuyt, L. M. Stals, and P. Moser, Phys. Rev. B 27, 5257 (1983).

[97] H. E. Schaefer, Phys. Status Solidi 102, 47 (1987). 
[98] H.-P. Scholz, Messungen der absoluten leerstellenkonzentration in nickel und geordneten intermetallischen nickellegierungen mit einem differentialdilatometer (Measurements of the absolute vacancy concentration in nickel and ordered intermetallic nickel alloys with a differential dilatometer), Ph.D. thesis, University of Göttingen, 2001.

[99] W. Wycisk and M. Feller-Kniepmeier, Phys. Status Solidi 37, 183 (1976).

[100] G. Michot and B. Deviot, Rev. Phys. Appl. 12, 1815 (1977).
[101] W. Wycisk and M. Feller-Kniepmeier, J. Nucl. Mater. 69, 616 (1978).

[102] A. Metsue, A. Oudriss, J. Bouhattate, and X. Feaugas, J. Chem. Phys. 140, 104705 (2014).

[103] R. Nazarov, T. Hickel, and J. Neugebauer, Phys. Rev. B 85, 144118 (2012).

[104] L. Ruch, D. R. Sain, H. L. Yeh, and L. Girifalco, Solid State Commun. 18, vi (1976).

[105] J. S. Wróbel, D. Nguyen-Manh, M. Y. Lavrentiev, M. Muzyk, and S. L. Dudarev, Phys. Rev. B 91, 024108 (2015). 